\title{
Comparison of Neural Network NARMA-L2 Model Reference and Predictive Controllers for Nonlinear EMS Magnetic Levitation Train
}

\author{
Mustefa Jibril ${ }^{1} \quad$ Eliyas Alemayehu Tadese ${ }^{2}$ \\ 1. Msc, Department of Electrical \& Computer Engineering, Dire Dawa Institute of Technology, Dire Dawa, \\ Ethiopia \\ 2. Msc, Faculty of Electrical \& Computer Engineering, Jimma Institute of Technology, Jimma, Ethiopia
}

\begin{abstract}
Magnetic levitation system is operated primarily based at the principle of magnetic attraction and repulsion to levitate the passengers and the train. However, magnetic levitation trains are rather nonlinear and open loop unstable which makes it hard to govern. In this paper, investigation, design and control of a nonlinear Maglev train based on NARMA-L2, model reference and predictive controllers. The response of the Maglev train with the proposed controllers for the precise role of a Magnetic levitation machine have been as compared for a step input signal. The simulation consequences prove that the Maglev teach system with NARMA-L2 controller suggests the quality performance in adjusting the precise function of the system and the device improves the experience consolation and street managing criteria.
\end{abstract}

Keywords: - Maglev train, NARMA-L2 controller, model reference controller, predictive controller

DOI: $10.7176 / \mathrm{IKM} / 10-4-03$

Publication date:May $31^{\text {st }} 2020$

\section{Introduction}

The MAGLEV system dynamics are strongly nonlinear, but they can be separated into mechanical dynamics and fast electrical dynamics. The most essential usage of magnetic levitation is in operation of magnetically levitated trains. Magnetically levitated trains are certainly the most advanced vehicles currently available to railway industries. Maglev is the primary fundamental innovation in the discipline of railroad technology since the invention of the railroad. Magnetically levitated control is a highly modern-day vehicle. Maglev automobiles use noncontact magnetic levitation, steerage and propulsion structures and don't have any wheels, axles and transmission. Contrary to standard railroad vehicles, there's no direct physical contact between maglev vehicle and its guide way. These vehicles circulate along magnetic fields which might be established between the car and its guide way. Conditions of no mechanical touch and no friction furnished by means of such generation makes it viable to attain better speeds of road attributed to such trains. Manned maglev motors have recorded velocity of road same to $581 \mathrm{~km} / \mathrm{hr}$. The alternative of mechanical additives of the way of wear-unfastened electronics overcomes the technical restrictions of wheel-on-rail generation. Application of magnetically levitated trains has attracted several transportation industries at some point of the sector. Magnetically levitated trains are the most current advancement in railway engineering especially in transportation industries. Maglev trains can be with no trouble considered as a solution for transportation needs of the modern time in addition to destiny desires of the world. There is form of designs for maglev structures and engineers keep revealing new thoughts about such systems.

These techniques nonetheless required using a linearized model of MAGLEV systems. There had been novel nonlinear control strategies without version linearization techniques. The stabilization place became notably widened by means of these nonlinear manage algorithms because the controller successfully eliminates or dominates the nonlinearities of MAGLEV system dynamics.

High Speed Maglev trains are carried out via the combos of magnetic field. This may be done by using Levitation and Propulsion magnets which might be embedded in tracks. The levitation magnets assist to levitate without any assist rater then magnetic fields whereas the propulsion magnets are chargeable for the movement of the trains. Guide methods are used to maintain the trains on course.

Maglev suspension systems are divided into 2 types: Electromagnetic Suspension (EMS) and Electrodynamics Suspension (EDS). There are sorts of vehicles which might be manufactured based totally on those two forms of systems. Basically, there are two foremost factors in a maglev system along with its car and the manual way. The 3 number one features in maglev generation are levitation, propulsion, and guidance. Magnetic forces perform all of those. Magnets are used to generate such magnetic forces. For EMS structures, these magnets are located within the vehicle while for EDS structures magnets are located inside the train.

Performance of EMS device is based on attractive magnetic forces, even as EDS device works with repulsive magnetic forces. In EDS machine, the automobile is levitated about 1 to ten $\mathrm{cm}$ above the guide way using repulsive forces. In EMS system, the vehicle is levitated approximately 1 to $2 \mathrm{~cm}$ above the guide way using 
attractive forces. In EMS system, the electromagnets on the vehicle engage with and are attracted to levitation rails at the guide way. Electromagnets attached to the train are directed up in the direction of the guide way, which levitates the train above the guide way and maintains the vehicle levitated. Control of allowed air gaps among the guide way and train is done by means of the usage of surprisingly advanced manipulate structures.

\section{Mathematical Models}

\subsection{Nonlinear Modelling of EDS Maglev Train}

In order to develop the Nonlinear Modelling of EDS Maglev Train, we need to develop the mathematical modelling of the Electromagnetic and Mechanical subsystems separately.

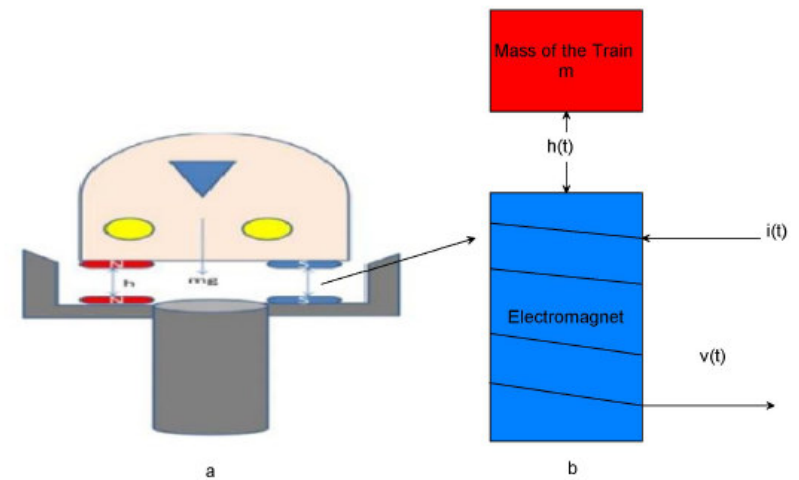

Figure 1: (a) EDS Model, (b) Single axis magnetic suspension system

Figure 1 shows a single axis magnetic levitation system is used, as well as electromagnetic and mechanical equations.

Apply Kirchhoff's voltage equation for the electric circuit

$$
V=V_{R}+V_{L} \Rightarrow u(t)=i R+L \frac{d i}{d t}
$$

Where $\mathrm{u}, \mathrm{I}, \mathrm{R}$ and $\mathrm{L}$ is applied voltage input, current in the electromagnet coil, coil's resistance and coil's inductance respectively.

Energy stored in the inductor can be written as

$$
W_{\text {IStored }}=\frac{1}{2} L i^{2}
$$

Since power in electrical system $(P e)=$ Power in the mechanical system $(P \mathrm{~m})$,

Where $P_{e}=\frac{d W_{\text {Isored }}}{d t}$ and $P_{m}=-f_{m} \frac{d x}{d t}$ therefore

$$
f_{m}=-\frac{d W_{\text {IStore }}}{d t} \frac{d t}{d x}=-\frac{d W_{\text {IStore }}}{d x}
$$

Where $f m$ is known as electromagnet force Now substituting (2) in the equation (3),

$$
\left.\begin{array}{l}
f_{m}=-\frac{d}{d x}\left(\frac{1}{2} L i^{2}\right) \\
=-\frac{1}{2} i^{2} \frac{d}{d x}(L)
\end{array}\right\}
$$

Since the inductance $L$ is a nonlinear function of train position (x) we shall neglect the leakage flux and eddy current effects (for simplicity), so that the inductance varies with the inverse of train position as follows:

$$
L=\frac{K}{x} \quad \text { Where in } K=\frac{\mu_{0} N^{2} A}{2}
$$

Where, $\mu 0$ is the inductance constant, $\mathrm{A}$ is the pole area, $\mathrm{N}$ is the number of coil turns and $k$ is electromagnet force constant. 


$$
\left.\begin{array}{c}
f_{m}=-\frac{1}{2} i^{2} \frac{d}{d x}\left(\frac{k}{x}\right) \\
=-\frac{1}{2} i^{2}\left(-\frac{k}{x^{2}}\right) \\
\therefore f_{m}=\frac{K}{2}\left(\frac{i^{2}}{x^{2}}\right)
\end{array}\right\}
$$

If $f m$ is electromagnetic force produced by input current, $f \mathrm{~g}$ is the force due to gravity and $f$ is net force acting on the train, the equation of force can be written as

$$
\left.\begin{array}{l}
f_{g}=f_{m}+f \\
=f_{m}+m\left(\frac{d^{2} x}{d t^{2}}\right) \\
\Rightarrow m \frac{d v}{d t}=f_{g}-f_{m}=m g-\frac{K}{2}\left(\frac{i(t)}{x(t)}\right)^{2}
\end{array}\right\}
$$

Where $m=$ train mass and $v=d \mathrm{x} / d t=d h / d t$, which is velocity of the train movement.

At equilibrium the force due to gravity and the magnetic force are equal and oppose each other so that the train levitates. i.e. $f \mathrm{~g}=-f m$ and $f=0$. On the basis of electro-mechanical modeling, the nonlinear model of magnetic levitation system can be described as follows:

The general form of an affine system

$$
\frac{d x}{d t}=f(x)+g(x) \cdot u
$$

Is obtained by denoting variables for state space representation as follows

$$
\left.\begin{array}{l}
x_{1}=h=x \\
x_{2}=\frac{d h}{d t}=v \\
x_{3}=i
\end{array}\right\}
$$

Substitute equation (9) or the state variables in to equation (1) and (7)

$$
\left.\begin{array}{l}
u(t)=x_{3} \cdot R+L \cdot \dot{x}_{3} \\
m \cdot \dot{x}_{2}=m \cdot g-\frac{K}{2}\left(\frac{x_{3}}{x_{1}}\right)^{2}
\end{array}\right\}
$$

Then the nonlinear state space model is

$$
\left.\begin{array}{l}
\dot{x}_{1}=x_{2} \\
\dot{x}_{2}=\left(g-\frac{K}{2 m}\right)\left(\frac{x_{3}}{x_{1}}\right)^{2} \\
\dot{x}_{3}=\frac{u}{L}-x_{3} \cdot \frac{R}{L}
\end{array}\right\}
$$

Nonlinear model in matrix form is given by

$$
\left(\begin{array}{c}
\dot{x}_{1} \\
\dot{x}_{2} \\
\dot{x}_{3}
\end{array}\right)=\left(\begin{array}{c}
x_{2} \\
\left(g-\frac{K}{2 m}\right)\left(\frac{x_{3}}{x_{1}}\right)^{2} \\
-\frac{R}{L} \cdot x_{3}
\end{array}\right)+\left(\begin{array}{c}
0 \\
0 \\
\frac{1}{L}
\end{array}\right) u
$$

Table 1: Physical paremeters of EMS Maglev train 


\begin{tabular}{|c|c|c|}
\hline Parameter & Unit & Value \\
\hline $\mathrm{M}$ & $\mathrm{kg}$ & 15,500 \\
\hline $\mathrm{R}$ & $\Omega$ & 50 \\
\hline $\mathrm{L}$ & $\mathrm{H}$ & 1 \\
\hline$i_{0}$ & $\mathrm{~A}$ & 200 \\
\hline$x_{0}$ & $\mathrm{~mm}$ & 26 \\
\hline $\mathrm{k}$ & $\frac{N m^{2}}{A^{2}}$ & 0.01 \\
\hline $\mathrm{g}$ & $\frac{m}{s^{2}}$ & 9.8 \\
\hline
\end{tabular}

\section{The Proposed Controller Design}

\subsection{NARMA-L2 Controller Design}

One of the main features of the NARMA-L2 neurocontroller is to transform nonlinear system dynamics into linear dynamics by canceling the nonlinearities. We begins by describing how the identified neural network model can be used to design a controller. The advantage of the NARMA-L2 form is that you can solve for the control input that causes the system output to follow a reference signal:

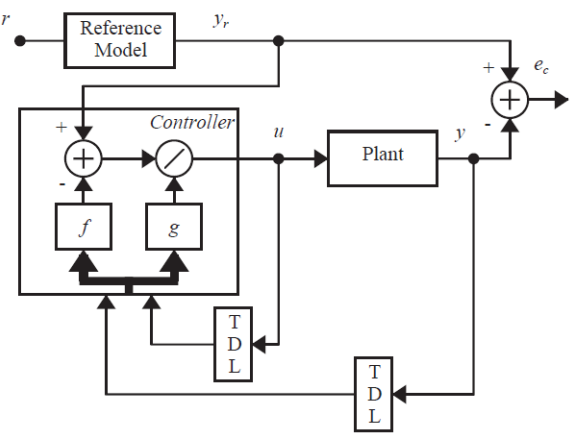

Figure 2 NARMA-L2 Controller.

\subsection{Model Reference Controller Design}

The model reference controller is designed to contain two neural networks: a neural network controller and a neural network plant model, as shown in Figure 3. The plant model is identified first, and then the controller is trained so that the plant output follows the reference model output

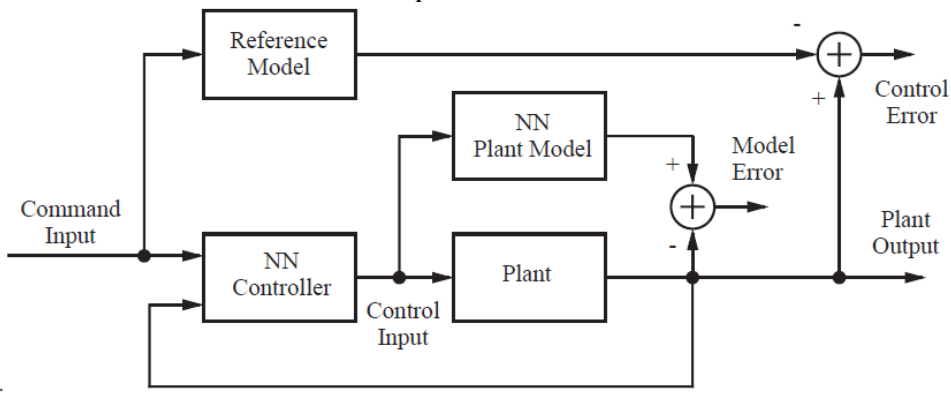

Figure 3 Model Reference Control Architecture

\subsection{Predictive Controller Design}

There are different types of neural network predictive controller that are based on linear model controllers. The proposed neural network predictive controller uses a neural network model of a nonlinear plant to predict future plant performance. The proposed controller then calculates the control input that will optimize plant performance over a specified future time horizon. The primary goal of the model predictive control is to determine the neural network plant model. Then, the plant model is used by the controller to predict future performance. The process is represented by Figure 4. 


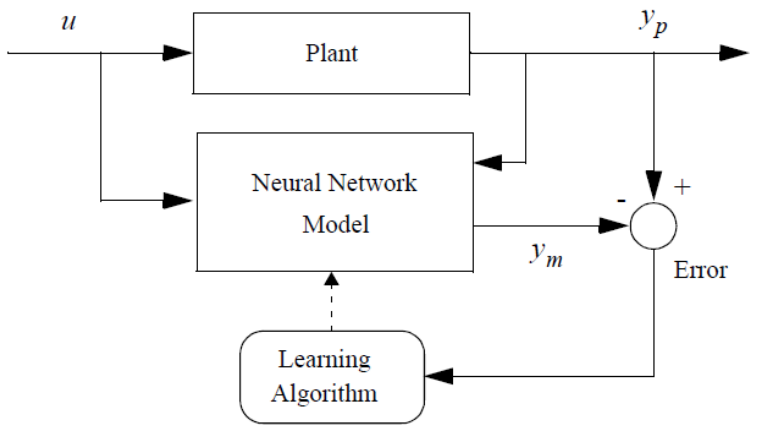

Figure 4 Plant Identification

\section{Result and Discussion}

\subsection{Maglev train system simulation response}

The simulation output for Maglev train system with NARMA-L2, model reference and predictive controllers for a Step input is shown in Figure 5.

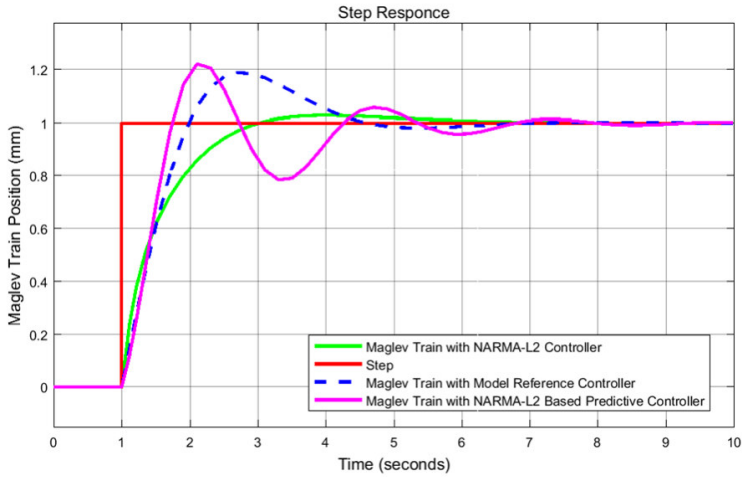

Figure 5 Maglev train system step response

4.2 Numerical values of the Performance of NARMA-L2, model reference and predictive Controllers The numerical values of the proposed controllers is shown in Table 2 below.

Table 2. Numerical values of the proposed controllers

\begin{tabular}{|c|c|c|c|}
\hline Controller & Rise time (sec) & Settling time (sec) & Percent Overshoot (\%) \\
\hline NARMA-L2 & 1.241 & 6.7 & 2.577 \\
\hline M Reference & 0.75 & 7 & 18.452 \\
\hline Predictive & 0.556 & 9 & 22.840 \\
\hline
\end{tabular}

\section{Conclusion}

Magnetic levitation system is designed and simulated using Matlab/Simulink successfully. The output of the magnetic levitation train is determined and analyzed.

The simulation result showed that the settling time of NARMA-L2 controller is smaller than the settling time of model reference and predictive controllers. The rise time of predictive controller is smaller than the rising time of NARMA-L2 and model reference controller. But the percentage overshoot of NARMA-L2 controller is very good when compared with model reference and predictive controllers. Finally the simulation result prove the effectiveness of the NARMA-L2 controller.

\section{References}

[1]. Asogwa Tochukwu et al. "Improving the Intelligent Control of Magnetic Levitation Ball using Artificial Neural Network" International Journal of Engineering and Computer Science, Vol. 8 No. 6, pp. 24679-24685, 2019.

[2]. J. Xie et al. "Research on the Sensetivity of Magnetic Levitation (Maglev) Devices" Journal of Magnetism and Magnetic Materials, Vol. 468, pp. 100-104, 2018.

[3]. Yang Y et al. "Study on the Optimization of Linear Induction Motor Traction System for Fast Speed Maglev Train" Journal of Transportation Systems and Technology, Vol. 4 No. 3, pp. 156-164, 2018.

[4]. Costa F. et al. "Characterization of Levitation Force for a Superconducting Magnetic Levitation Vehicle" Journal of Transportation Systems and Technology, Vol. 4 No. 3, pp. 124-133, 2018. 
[5]. Long Z. et al “A Novel Design of Electromagnetic Levitation System for a High Speed Maglev Train” Journal of Transportation Systems and Technology, Vol. 4 No. 3, pp. 212-224, 2018.

[6]. Zhizhou Zhang et al. "Real-Time Adaptive Control of a Magnetic Levitation System with a Large Range of Load Disturbance" Sensors (Basel), Vol. 18, Issue. 5, 2018. 